Check for updates

Cite this: RSC Adv., 2018, 8, 33534

Received 17th August 2018

Accepted 11th September 2018

DOI: 10.1039/c8ra06880a

rsc.li/rsc-advances

\section{Heterogeneous catalytic ozonation of ciprofloxacin in aqueous solution using a manganese-modified silicate ore $\uparrow$}

\author{
Lisha Luo, (D) ab Donglei Zou, ${ }^{a}$ Dongwei Lu, (D) *c Bingjing Xin, (DD ${ }^{\mathrm{b}}$ Ming Zhou, ${ }^{\mathrm{b}}$ \\ Xuedong Zhail and Jun Ma*c
}

\begin{abstract}
Manganese modified silicate ore (MnSO) prepared using an impregnation method was used as a heterogeneous ozonation catalyst, and the catalytic activity was evaluated by the degradation of ciprofloxacin (CIP). The results showed that the manganese oxide was successfully loaded onto natural silicate ore (SO). The degradation and mineralization efficiencies of CIP were considerably improved in the presence of MnSO. Under optimal conditions, the CIP removal process followed the pseudo-firstorder reaction model well. The degradation rate constant of $\mathrm{MnSO} / \mathrm{O}_{3}$ was 1.7 times and 3.3 times higher than those of $\mathrm{SO} / \mathrm{O}_{3}$ and only $\mathrm{O}_{3}$, respectively. During the ozonation of the $\mathrm{CIP}$ aqueous solution in the presence of $\mathrm{MnSO}$, the TOC removal rate reached $61.2 \%$ at $60 \mathrm{~min}$, but was only $30.8 \%$ using ozonation alone. The addition of tert-butanol (TBA) significantly inhibited the degradation efficiency of $\mathrm{CIP}$, which indicated that catalytic ozonation of $\mathrm{MnSO}$ followed a hydroxyl radical $(\cdot \mathrm{OH})$ reaction mechanism. Furthermore, MnSO showed great stability and durability over several reaction cycles.
\end{abstract}

\section{Introduction}

In recent years, antibiotics have been widely developed and increasingly used in the aquaculture, animal farming, and human pharmaceutical industries. ${ }^{\mathbf{1}, 2}$ Most antibiotics enter aquatic systems and lead to chemical pollution and resistant gene pollution. ${ }^{3}$ Ciprofloxacin (CIP), as the second generation of fluoroquinolones, has been extensively used because of its capacity for efficient disease control and enhancing animal growth. ${ }^{4,5}$ The level of CIP concentration detected in the environment has increased from $\mathrm{ng} \mathrm{\textrm {L } ^ { - 1 }}$ to $\mathrm{mg} \mathrm{L}^{-1} \cdot{ }^{6}$ Conventional biological wastewater treatment processes used in sewage treatment plants (STPs) are not effective for the removal of CIP because of its poor biodegradability. ${ }^{7}$ Therefore, it is urgent and necessary to develop an effective technology to remove CIP.

Heterogeneous catalytic ozonation, which combines ozone with a solid catalyst, is an effective advanced oxidation technology for the degradation and mineralization of CIP. ${ }^{8-11}$ The solid catalyst can accelerate the decomposition of ozone to hydroxyl radical $(\cdot \mathrm{OH})$ and can also adsorb organic pollutants

${ }^{a}$ Key Laboratory of Ministry of Education for Groundwater Resources and Environment, College of New Energy and Environment, Jilin University, Changchun 130000, P. R. China

${ }^{b}$ Jilin Institute of Chemical Technology, Jilin 130022, P. R. China

${ }^{c}$ State Key Laboratory of Urban Water Resource and Environment, Harbin Institute of Technology, Harbin 150090, P. R. China.E-mail: lvdongwei@hit.edu.cn

$\dagger$ Electronic supplementary information (ESI) available. See DOI: 10.1039/c8ra06880a on its surface to accelerate subsequent reactions between the - $\mathrm{OH}$ and pollutant. ${ }^{8}$ Manganese oxide is among the most widely studied metal oxides as an ozonation catalyst because of its unique characteristics, such as environmental friendliness, diverse crystallographic structure, facile fabrication, and abundance in soil. ${ }^{12-14}$ As demonstrated in this study, manganese oxide supported by various porous materials (carbonbased multi-pore materials, ${ }^{15,16}$ honeycomb ceramics, ${ }^{17}$ and mesoporous molecular sieve ${ }^{18}$ ) can effectively enhance mechanical performance and provide more catalytic active sites. In addition, porous materials supported by manganese oxide can be easily separated, avoiding the waste of catalyst and secondary pollution. However, the carbon-based material is easily oxidized by ozone and can generate a large quantity of residual sludge that needs to be processed. ${ }^{19-21}$ In addition, the production of synthetic materials may cause certain pollution problems to the environment and increase costs. ${ }^{22}$ These disadvantages limit the application of carbon-based materials and synthetic catalysts in full-scale systems.

Natural mineral materials have been reported as heterogeneous ozone catalysts or supports because they are environmentally friendly and of low cost. Previous studies have indicated that natural mineral materials and their modified forms have been investigated for their potential to catalyze the ozonation of various organic contaminants in water and wastewater including bauxite, ${ }^{21}$ magnetite ore, ${ }^{22}$ brucite, ${ }^{23}$ goethite, ${ }^{24}$ and perovskite. ${ }^{25}$ However, the specific surface area of some natural mineral materials is small, and the adsorption performance of the target materials is poor $;^{\mathbf{2 1 - 2 5}}$ thus, they 
provide limited catalytic active sites. Therefore, it is necessary to seek a natural mineral material that simultaneously possesses a large surface area and a strong adsorption capacity for catalytic ozonation of pollutants.

In this study, we used a type of natural silicate ore as the support to fabricate a composite catalyst. $\mathrm{MnO}_{x}$ was selected as the active component of the catalyst. A composite catalyst was prepared using the impregnation method, and the MnSO obtained was characterized using different techniques. The influence of catalytic dose, solution $\mathrm{pH}, \mathrm{CIP}$ concentration, and the reaction temperature on the degradation and mineralization of CIP were studied. Furthermore, a possible reaction mechanism for ozonation of CIP with MnSO is discussed.

\section{Materials and methods}

\subsection{Chemicals}

Silicate ore was obtained from advanced oxidation technology research center of the Harbin Institute of Technology. CIP was purchased from Sigma-Aldrich ( $\geq 98 \%)$. Manganese nitrate was purchased from the Tianjin Baishi Chemical Reagent Co., Ltd, tert-butyl alcohol (TBA) was purchased from the Tianjin Ruijinte Chemical Reagent Co., Ltd. Sodium thiosulphate was supplied by the Tianjin Reagent Chemical Co., Ltd. All chemicals used in this study were analytic grade and used without further purification. Deionized water was used during the experiment. The $\mathrm{pH}$ of the solution was adjusted with nitric acid and sodium hydroxide.

\subsection{Preparation of MnSO catalysts}

Modified natural silicate ore was prepared using an impregnation method. Detailed preparation information is as follows. First, the natural silicate ore particles were washed with deionized water several times to remove surface dust, and then grinded to $60-80$ mesh through a high-speed universal mill. Second, $5 \mathrm{~g}$ of crushed natural silicate ore was soaked in $\mathrm{Mn}\left(\mathrm{NO}_{3}\right)_{2}\left(0.5 \mathrm{~mol} \mathrm{~L}{ }^{-1}, 100 \mathrm{~mL}\right)$ solution, and oscillated at a constant speed $(120 \mathrm{rpm})$ for $24 \mathrm{~h}$ at $30{ }^{\circ} \mathrm{C}$. Following precipitation and filtration, the silicate ore was dried at $70{ }^{\circ} \mathrm{C}$ for $36 \mathrm{~h}$ before calcination treatment at $500{ }^{\circ} \mathrm{C}$ for $5 \mathrm{~h}$ in air. Then, the MnSO samples were cooled to room temperature and stored in a desiccator before use.

\subsection{Catalyst characterization}

Scanning electron microscopy (SEM, Hitachi SU8000, Japan) with an acceleration voltage of $15 \mathrm{kV}$ was used for analyzing the morphology of the samples, and the catalyst surface was sputter coated with gold. The crystalline shape of the catalyst was analyzed using X-ray diffraction (XRD, D/max-RA, Rigaku), operating with a $\mathrm{Cu}$ anode at $40 \mathrm{kV}$ and $100 \mathrm{~mA}$. The 2 theta data from $10^{\circ}$ to $80^{\circ}$ were obtained at a scanning speed of $0.03^{\circ} \mathrm{s}^{-1}$. The specific surface area, total pore volume, and average pore size of the catalysts were measured using a nitrogen gas adsorption analyzer (ASAP-2020, Micromeritics, USA) based on the Brunauer-Emmett-Teller (BET) method. Infrared spectra of the catalysts were measured under ambient conditions using a Fourier transform infrared (FT-IR) spectrum instrument (Spectrum 2000, Perkin Elmer, USA). X-ray photoelectron spectroscopy (XPS, Kratos-AXIS ULTRA DLD) using Al $\mathrm{K} \alpha$ as the source was used to determine the surface components of the composite. The characterization results of SEM, XRD, BET and FT-IR are provided in ESI (Fig. S1-S4 and Table S1 †).

\subsection{Catalytic ozonation procedure}

Batch catalytic ozonation degradation experiments of CIP were conducted in a $500 \mathrm{~mL}$ flat bottom beaker. A microporous titanium diffuser was connected at the bottom of the reactor to diffuse the ozone gas entering the reactor. Ozone was produced via a DHX-I ozone generator (Harbin Jiujiu Electrochemistry Technology Co., Ltd., China). The inlet ozone gas concentration was approximately $0.4 \mathrm{mg} \mathrm{min}{ }^{-1}$ and the ozone flow rate was

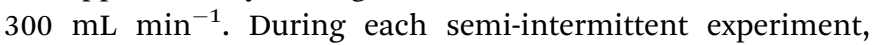
$400 \mathrm{~mL}$ of CIP aqueous solution $\left(20 \mathrm{mg} \mathrm{L}^{-1}\right)$ and a certain quality catalyst were added to the beaker. During the whole process, the slurry was continuously stirred to ensure that the catalyst was completely fluidized in the reaction solution. The experiment was conducted at $20^{\circ} \mathrm{C}$ using a cryostat. The water samples were collected at regular intervals and filtered through cellulose acetate filters $(0.22 \mu \mathrm{m})$ before analysis of CIP and total organic carbon (TOC) concentrations. A small amount of sodium thiosulfate solution was added as the termination agent. To test the stability of the MnSO, the catalyst was collected by centrifugal separation after the catalytic ozonation reaction, washed several times with deionized water, and dried at $70^{\circ} \mathrm{C}$ for $24 \mathrm{~h}$ before reuse. The $\mathrm{pH}$ value of the solution was regulated using normal $\mathrm{HNO}_{3}$ and $\mathrm{NaOH}$ in all reactions for a comparison. To obtain effective experimental data, the experiment was repeated three times, and the results were averaged.

\subsection{Analysis methods}

CIP concentration was analyzed using high-performance liquid chromatography (HPLC; Agilent 1200 Series, DAD detector and XDB-C18 column) with a UV detector at $276 \mathrm{~nm}$. The mobile phase was acetonitrile and $0.1 \%$ formic acid $(88: 12, \mathrm{v} / \mathrm{v})$ with a $1.0 \mathrm{~mL} \mathrm{~min}^{-1}$ flow rate.

The first-order model was used to simulate the degradation kinetics of CIP, $C_{t} / C_{0}=\exp (-k t)$. Here, $k\left(\mathrm{~min}^{-1}\right)$ is the pseudofirst-order rate constant of CIP; $t$ ( $\mathrm{min}$ ) is the reaction time; and $C_{0}$ and $C_{t}$ denote the CIP concentrations in the solution at the initial concentration and a specific time point, respectively.

Total organic carbon (TOC) was measured using a total organic carbon analyzer. (TOC-VCPH, Shimadzu Japan). The concentration of leaching manganese was measured using inductively coupled plasma (ICP) (Perkin-Elmer optima 5300DV).

\section{Results and discussion}

\subsection{Characterization of catalysts}

The morphologies of the SO and MnSO were observed by scanning electron microscope (Fig. S1 $\dagger$ ). As shown in Fig. S1a, $\uparrow$ 

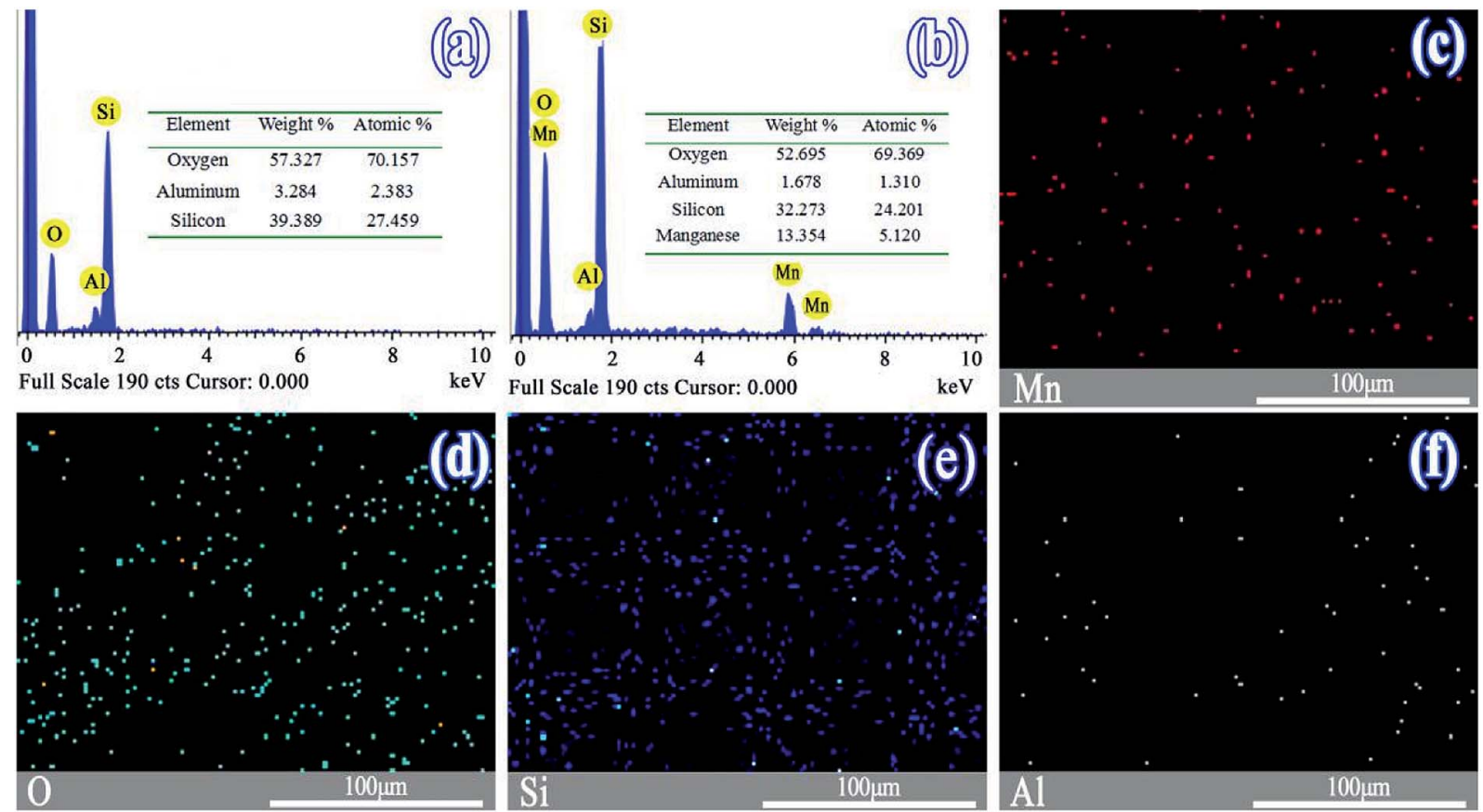

Fig. 1 EDS spectrum of (a) SO, (b) MnSO, and (c)-(f) EDS mapping images of the MnSO.

the surface of the SO was rough and uneven. Many particles were piled atop one another and interrelated. Pores were abundant. As shown in Fig. S1b, $\dagger$ the surface of the MnSO gradually smoothed with the $\mathrm{MnO}_{x}$ introduction, probably owing to the $\mathrm{MnO}_{x}$ on the surface of the SO.

Fig. 1a and $b$ show the EDS images of the SO and MnSO. The results confirmed the presence of the Mn element in the MnSO. The mass ratio of the surface $\mathrm{Mn}$ element for $\mathrm{MnSO}$ was $13.35 \%$. Based on the EDS mapping image as shown in Fig. $2 \mathrm{c}$ the manganese was uniformly distributed on the MnSO surface. The formation of metal oxides and their effective distribution play an important role in MnSO degrading refractory organic pollutants. ${ }^{26}$ The results indicated that the Mn element was successfully doped in the SO catalyst. XRD of the SO and MnSO sample is shown in Fig. S2. $\dagger$ In the pattern of the SO, the diffraction peaks matched with the published XRD pattern of the cristobalite (JCPDS no. 89-3607) and $\alpha$-quartz (JCPDS no. 851054). Compared to the SO, there were some unique diffraction peaks $\left(2 \theta=28.8^{\circ}, 37.5^{\circ}, 56.8^{\circ}, 32.9^{\circ}\right.$ and $\left.55.1^{\circ}\right)$ which corresponded to the (110), (101), (221), (222), and (440) faces, respectively, on the MnSO sample. These diffraction peaks belong to $\mathrm{MnO}_{2}$ and $\mathrm{Mn}_{2} \mathrm{O}_{3} \cdot{ }^{27,28}$ Therefore, it can be deduced that the $\mathrm{MnO}_{x}$ were effectively loaded on the SO surface.

The surface elemental composition and chemical states of the SO and MnSO were further investigated using XPS. As shown in Fig. 2a, compared to the characteristic peaks of the $\mathrm{SO}$, there not only were characteristic peaks corresponding to $\mathrm{Si}$ 2p, C 1s, O 1s and Al 2p, but also to Mn 2p in the XPS spectrum of the MnSO composites, which confirms the presence of $\mathrm{Mn}$ in the MnSO composites. Fig. 2b shows the peak at $102.9 \mathrm{eV}$ corresponding to Si $2 \mathrm{p} .{ }^{29} \mathrm{Fig}$. $2 \mathrm{c}$ shows the spectrum of $\mathrm{Mn} 2 \mathrm{p}_{3 / 2}$ shows three peaks, corresponding to $\mathrm{MnO}_{2}$ (641.1 and $643.4 \mathrm{eV}$ ) and $\left.\mathrm{Mn}_{2} \mathrm{O}_{3}(642.2 \mathrm{eV})\right)^{30,31}$ which were consistent with the aforementioned XRD results. This indicated that the $\mathrm{MnO}_{2}$ and $\mathrm{Mn}_{2} \mathrm{O}_{3}$ were successfully doped into the SO. As shown in Fig. 2d, the $\mathrm{O} 1 \mathrm{~s}$ peaks at $530.7 \mathrm{eV}, 531.3 \mathrm{eV}, 532.7 \mathrm{eV}$, and $531.9 \mathrm{eV}$ were assigned to the $\mathrm{Mn}-\mathrm{O}, \mathrm{Al}-\mathrm{O}, \mathrm{Si}-\mathrm{O}$, and $\mathrm{OH}(\mathrm{ads})$, respectively. The $\mathrm{OH}(\mathrm{ads})$ is beneficial for the generation of $\cdot \mathrm{OH}$ in catalytic ozonation. ${ }^{32}$

As shown in Fig. $\mathrm{S} 3, \dagger$ the $\mathrm{N}_{2}$ adsorption-desorption isotherms of the SO and MnSO were both identified as type IV according to the International Union of Pure and Applied Chemistry classification. This means that the SO and MnSO

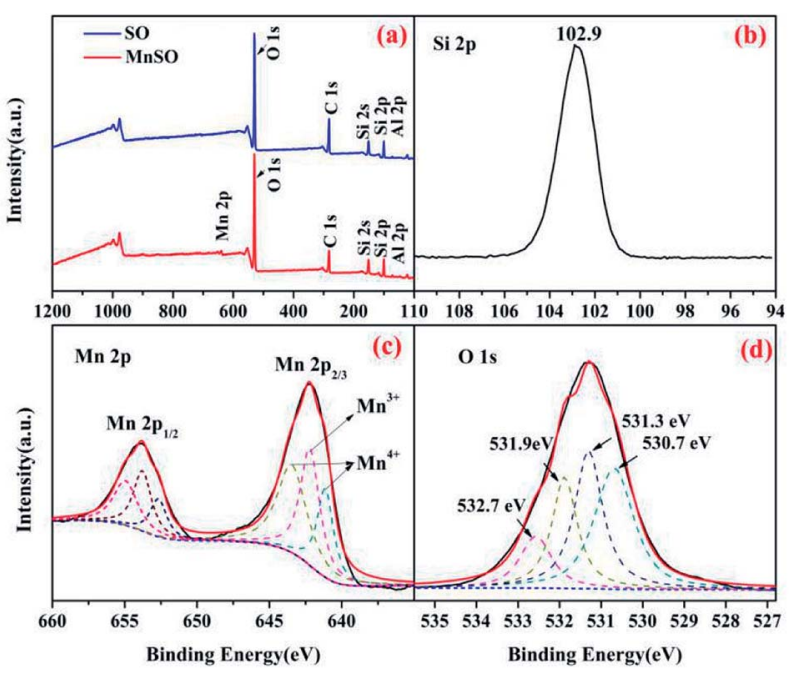

Fig. 2 XPS spectra analysis of the SO and MnSO catalyst. (a) Widerange scan of the SO and MnSO; (b) Si 2p, (c) Mn 2p, and (d) O 1s of the MnSO composites. 
catalyst surface were mesoporous structures. ${ }^{33}$ The BET of both the SO and MnSO are listed in Table S1. $\dagger$ The specific surface areas of the $\mathrm{SO}$ and $\mathrm{MnSO}$ composite were determined to be 75.56 and $57.82 \mathrm{~m}^{2} \mathrm{~g}^{-1}$, respectively. The results showed that the loading of $\mathrm{MnO}_{x}$ resulted in a decrease in the average pore volume, specific surface area, and pore size of the SO. This illustrated that the manganese oxide dispersed into the pores of the SO and blocked them.

The FT-IR spectra were measured to investigate the surface function groups of the SO and MnSO. As shown in Fig. S4, $\dagger$ the absorption peak at $1105 \mathrm{~cm}^{-1}$ originated from $\mathrm{Si}-\mathrm{O} .^{21}$ The bands at $478 \mathrm{~cm}^{-1}$ were attributed to the bending and stretching modes of $\mathrm{AlO}_{6} \cdot{ }^{34}$ The presence of water molecules was supported by the appearance peaks of the bending vibration absorption of $\mathrm{H}-\mathrm{O}-\mathrm{H}$ at $1635 \mathrm{~cm}^{-1}$ and the stretching mode stretching vibration of $\mathrm{O}-\mathrm{H}$ at $3419 \mathrm{~cm}^{-1} \cdot{ }^{35,36}$

\subsection{Adsorption and catalytic ozonation of CIP by MnSO}

To evaluate the catalytic activity of the catalyst, the degradation and mineralization of CIP under different experimental conditions including adsorption, single ozonation, and catalytic ozonation experiments were determined as shown in Fig. 3. After $30 \mathrm{~min}$, the adsorption efficiencies in the presence of $\mathrm{SO}$ and MnSO were approximately $25.3 \%$ and $17.5 \%$, respectively. This type of natural SO and MnSO had good adsorption performance for the CIP, which benefited further ozonation reactions. The degradation of CIP reached $53 . \%$ over $30 \mathrm{~min}$ using a single ozonation process, whereas it increased to $74.9 \%$ and $92.1 \%$ by adding SO and MnSO during the catalytic ozonation process, respectively. The results showed that MnSO was an effective heterogeneous ozonation catalyst, which increased the degradation efficiency of CIP by approximately $39.1 \%$ as compared to the single ozonation process. As shown in Table $\mathrm{S} 2, \uparrow$ the CIP degradation rates by adsorption, single ozonation, and catalytic ozonation followed the pseudo-first-order kinetic model. The CIP degradation rate constant with $\mathrm{MnSO} / \mathrm{O}_{3}$ was 1.7 times higher than that of $\mathrm{SO} / \mathrm{O}_{3}$, and 3.3 times higher than that of single ozonation. The results show that MnSO enhanced the catalytic activity in the ozonation of CIP. In comparison with the catalyst of $\mathrm{MnO}_{x} / \mathrm{MNCNT}^{16}$ and $\mathrm{MnO}_{x} / \mathrm{MCM}-41$ (ref. 18) for refractory pollutant oxidative degradation, MnSO in this work

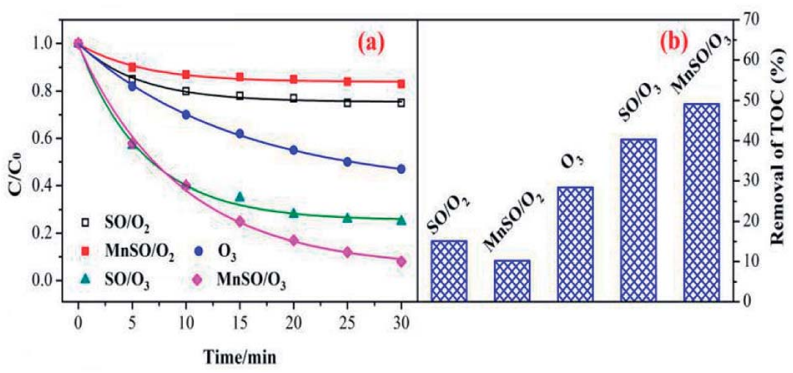

Fig. 3 Degradation and mineralization of CIP by ozonation alone and with MnSO. Experimental conditions: $\mathrm{pH}=7.0 ; \mathrm{C}_{0}=20 \mathrm{mg} \mathrm{L}^{-1}$; ozone gas flow rate $=0.3 \mathrm{~L} \mathrm{~min}^{-1}$; ozone gas concentration $=0.4 \mathrm{mg} \mathrm{min}^{-1}$; catalyst dose $=0.5 \mathrm{~g} \mathrm{~L}^{-1} ; T=20^{\circ} \mathrm{C}$. was relatively high efficient, widespread, easily prepared and low cost, which has a better application prospect.

In addition, the TOC removal efficiency obtained over $60 \mathrm{~min}$ during the MnSO catalytic ozonation process (61.2\%) was significantly higher than that during the SO catalytic ozonation (53.6\%) and single ozonation (30.8\%) processes. The adsorption process still had a great effect on the removal of TOC. The improvement of CIP degradation and TOC removal may have been a result of the following. First, the MnSO catalyst had a good adsorption capacity which could accelerate subsequent reactions between the ozone and pollutant. ${ }^{37}$ Second, the presence of the MnSO catalyst could promote decomposition of ozone to a hydroxyl radical $(\cdot \mathrm{OH}) .{ }^{8}$ The $\cdot \mathrm{OH}$ was non-selective and could enhance the CIP and TOC removal. However, the removal rate of TOC was significantly lower than that of the degradation of CIP. This result was because a large number of intermediates produced during the CIP degradation process were difficult to degrade via ozone. Although CIP can be largely removed, the intermediate products cannot be completely converted into $\mathrm{CO}_{2}$ and $\mathrm{H}_{2} \mathrm{O}$, thus reducing the removal effect of the TOC.

\subsection{Influence of the catalyst dosage on the catalytic ozonation process}

The catalyst dosage relates to the reaction rate of the ozone with an organic reaction, and also relates to the water treatment cost in practical application. Therefore, it is necessary to discuss the optimum amount of catalyst. As shown in Fig. 4, the degradation and mineralization efficiency of CIP significantly increase with an increase in catalyst dose. The CIP removal efficiencies improved from $82 \%$ to $92.5 \%$ and the removal efficiency of the TOC increased by $20 \%$.

The CIP degradation kinetic constants during the $\mathrm{MnSO} / \mathrm{O}_{3}$ process were enhanced from $0.058 \mathrm{~min}^{-1}$ to $0.094 \mathrm{~min}^{-1}$ as the catalyst dosage increased from $0.2 \mathrm{~g} \mathrm{~L}^{-1}$ to $1.0 \mathrm{~g} \mathrm{~L}^{-1}$. Simultaneously, the TOC removal rate constants by $\mathrm{MnSO} / \mathrm{O}_{3}$ also increased from $0.007 \mathrm{~min}^{-1}$ to $0.014 \mathrm{~min}^{-1}$ with the catalyst dosage increasing from $0.2 \mathrm{~g} \mathrm{~L}^{-1}$ to $1.0 \mathrm{~g} \mathrm{~L}^{-1}$. These results indicated that increasing the amount of catalyst in the solution was conducive to improving the specific surface area of the catalyst in the solution and facilitating decomposition of ozone

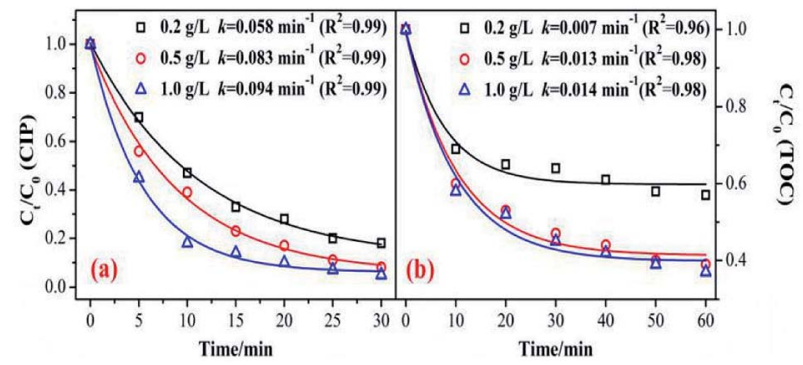

Fig. 4 Effect of catalyst dosage on the removal of CIP and TOC. (Experimental conditions: $\mathrm{pH}=7.0 ; \mathrm{C}_{0}=20 \mathrm{mg} \mathrm{L}^{-1}$; ozone gas flow rate $=0.3 \mathrm{~L} \mathrm{~min}-1$; ozone gas concentration $=0.4 \mathrm{mg} \mathrm{min}^{-1} ; T=$ $\left.20^{\circ} \mathrm{C}\right)$. 
molecules into more $\cdot \mathrm{OH}^{38}$ However, the removal efficiency and mineralization efficiency of CIP were not significantly improved when the addition of the catalyst was $1.0 \mathrm{~g} \mathrm{~L}^{-1}$ because of the limited amount of ozone in the system. In addition, when the catalytic dose continued to increase, the particles collided with each other, thus affecting the effective contact between the catalyst and ozone.

\subsection{Influence of CIP initial concentration on catalytic ozonation process}

Fig. 5 shows the effect of the initial concentration on the CIP and TOC removal during the MnSO catalytic ozonation process. It can be seen that the CIP or TOC removal decreased with increasing initial concentration. The CIP degradation kinetic constant was $0.104 \mathrm{~min}^{-1}$ with the initial concentration of $10 \mathrm{mg} \mathrm{\textrm {L } ^ { - 1 }}$, and it was approximately twice that with an initial concentration of $40 \mathrm{mg} \mathrm{L}{ }^{-1}$. This result indicates that when the initial concentration of CIP was low, the amount of dissolved ozone in the solution and the amount of $\cdot \mathrm{OH}$ from the decomposition of ozone in the solution were high. They can rapidly degrade CIP and also result in a higher mineralization effect. When the initial concentration of CIP increases, the ozone is rapidly utilized by the high concentration of CIP. At the same time, the intermediate product competes with ozone for CIP, thus the degradation effect of CIP was significantly reduced. ${ }^{39}$

The removal rate of the TOC corresponded to the trend in the catalytic ozonation degradation rate of CIP, but it was still much lower than that of the CIP degradation. It can be inferred that the speculation produced a large number of intermediates that were more resistant to ozonation than CIP.

\subsection{Influence of reaction temperature on the catalytic ozonation process}

Fig. 6 shows the influence of reaction temperature $(10,20$, and $35^{\circ} \mathrm{C}$ ) on the catalytic activity of the MnSO for ozonation of CIP. As shown in Fig. 6, when the reaction temperature increased from $10{ }^{\circ} \mathrm{C}$ to $20^{\circ} \mathrm{C}$, the degradation efficiencies of CIP and TOC greatly increased from $82.3 \%$ to $92.1 \%$ and 33.1 to $61.3 \%$, respectively. The kinetic information for CIP degradation and mineralization is shown in Fig. 6. It shows that when the

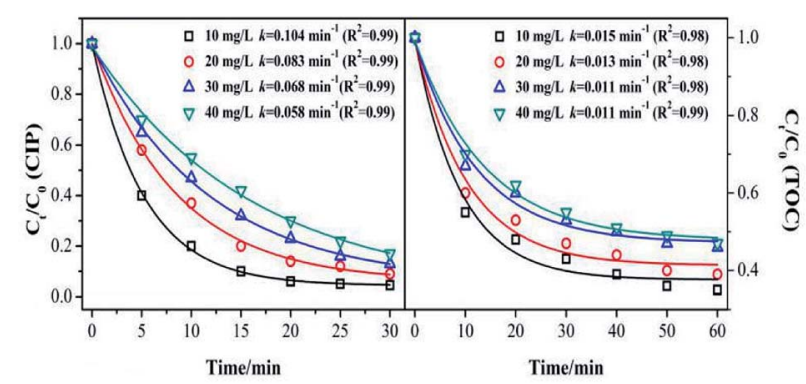

Fig. 5 Effect of initial concentration of the target on removal of CIP and TOC. (Experimental conditions: $\mathrm{pH}=7.0$; Ozone gas flow rate $=$ $0.3 \mathrm{~L} \mathrm{~min}-1$; ozone gas concentration $=0.4 \mathrm{mg} \mathrm{min}^{-1}$; catalyst dose $=$ $0.5 \mathrm{~g} \mathrm{~L}^{-1} ; T=20^{\circ} \mathrm{C}$ ).

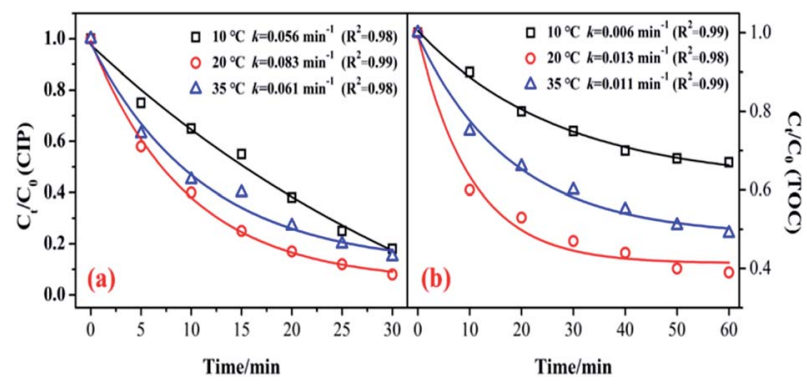

Fig. 6 Effect of reaction temperature on the removal of CIP and TOC. (Experimental conditions: $\mathrm{pH}=7.0 ; \mathrm{C}_{0}=20 \mathrm{mg} \mathrm{L}^{-1}$; ozone gas flow rate $=0.3 \mathrm{~L} \mathrm{~min}-1$; ozone gas concentration $=0.4 \mathrm{mg} \mathrm{min}^{-1}$; catalyst dose $=0.5 \mathrm{~g} \mathrm{~L}^{-1}$ ).

temperature was $20{ }^{\circ} \mathrm{C}$, the degradation rate was the fastest. However, when the temperature continued to increase to $35^{\circ} \mathrm{C}$, the removal efficiency was suppressed. Generally, a higher reaction temperature would lead to a faster reaction rate for a chemical reaction and mass transfer. In addition, a temperature increase can accelerate the decomposition of ozone and produce more hydroxyl radicals, which is beneficial to improving the catalytic ozonation effect. Nonetheless, the solubility of the ozone in water could decrease at higher temperatures. During the process of catalytic ozonation, the increase in reaction temperature results in these two opposing effects. ${ }^{\mathbf{4 0 , 4 1}}$ Therefore, the reaction temperature of $20^{\circ} \mathrm{C}$ has a positive effect on CIP degradation and mineralization during the process of ozonation of MnSO.

\subsection{Influence of initial $\mathrm{pH}$ on the catalytic ozonation process}

In the catalytic ozonation system, the initial $\mathrm{pH}$ value was the key factor that affected the ozone decomposition rate and hydroxyl formation..$^{\mathbf{8} 41}$ In addition, the charge of the organic compounds in the solution varied under different $\mathrm{pH}$ conditions. ${ }^{40,41}$ Fig. 7 shows the influence of the initial $\mathrm{pH}$ value on the CIP degradation and TOC removal efficiencies in catalytic ozonation. As shown in Fig. 7, the CIP removal efficiency clearly improved with an increase in the initial $\mathrm{pH}$ from $3.7(79.5 \%)$ to $10.3(95.8 \%)$. This was because in the alkaline solution more - $\mathrm{OH}$ were yielded which was beneficial to organic decomposition. ${ }^{8,42}$ A similar trend was found in the TOC removal. At initial $\mathrm{pH}$ values of $3.7,5.2,7,8.5$, and 11.3 , the TOC removals were $44.5 \%, 52.7 \%, 61.5 \%, 65.4 \%$, and $73.2 \%$ after $60 \mathrm{~min}$. The CIP

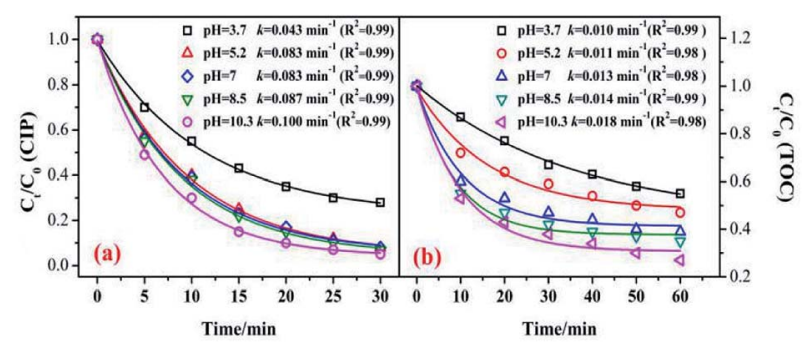

Fig. 7 Effect of $\mathrm{pH}$ on the removal of CIP and TOC. (Experimental conditions: $C_{0}=20 \mathrm{mg} \mathrm{L}^{-1}$; ozone gas flow rate $=0.3 \mathrm{~L} \mathrm{~min}^{-1}$; ozone gas concentration $=0.4 \mathrm{mg} \mathrm{min}^{-1}$; catalyst dose $=0.5 \mathrm{~g} \mathrm{~L}^{-1} ; T=$ $\left.20^{\circ} \mathrm{C}\right)$. 
degradation kinetic constants of the $\mathrm{MnSO} / \mathrm{O}_{3}$ process were enhanced from $0.0043 \mathrm{~min}^{-1}$ to $0.100 \mathrm{~min}^{-1}$ as the $\mathrm{pH}$ increased from 3.7 to 10.3. Simultaneously, the TOC removal rate constants by $\mathrm{MnSO} / \mathrm{O}_{3}$ also increased from $0.010 \mathrm{~min}^{-1}$ to $0.018 \mathrm{~min}^{-1}$ as the $\mathrm{pH}$ increased from 3.7 to 10.3.

Therefore, even though the intermediates of CIP were resistant to ozonation, they could be further oxidized or even mineralized by $\cdot \mathrm{OH}$ generated from the $\mathrm{O}_{3}$ decomposition. The increased production of $\cdot \mathrm{OH}$ during ozonation was an effective method to improve the TOC removal.

\subsection{Catalyst stability and reusability}

During the application process of the catalyst, the catalyst may have some problems such as the dissolution of the active component and damage to the active sites, which can lead to a significant decrease in its activity. Therefore, in the heterogeneous catalytic ozonation system, among the important measures to evaluate the effect of the catalyst was its reuse rate. Recycling experiments were conducted to evaluate the activity and stability of MnSO catalyst.

As shown in Table 1, the CIP and TOC removal efficiency slightly decreased with the increase in the catalyst reuse times. CIP degradation was reduced by $c a$. $3.0 \%$ and the TOC removal by $c a .2 .9 \%$. It was found that after four times of reuse, the MnSO catalyst still had good catalytic activity on CIP degradation and mineralization. The concentration of the released manganese ions in solution repeated over four times were detected to be $0.097 \mathrm{mg} \mathrm{L}^{-1}, 0.088 \mathrm{mg} \mathrm{L}^{-1}, 0.076 \mathrm{mg} \mathrm{L}^{-1}$, and $0.069 \mathrm{mg} \mathrm{L}^{-1}$, respectively. Although slight leaching of manganese ions was found, the catalytic capacity of the MnSO was hardly affected. The dissolution concentration of manganese ions was less than the limit in the drinking water of China $\left(0.1 \mathrm{mg} \mathrm{L}{ }^{-1}\right) .{ }^{43}$ Thus, it had less effect on the safety of drinking water. We have characterized the catalyst by XRD after reactions (Fig. S5 $\dagger$ ). The results showed that the diffraction peaks of the catalyst remained unchanged before and after the reaction. This further demonstrated the good stability of the catalyst. Therefore, it could be concluded that the MnSO had great catalytic activity and stability, and is a promising catalyst for the removal of antibiotic wastewater.

\subsection{Possible reaction mechanism during the MnSO process}

3.8.1. Radical scavenging experiment. TBA is a well-known hydroxyl radical scavenger that can capture hydroxyl radicals

Table 1 Stability and activity of the MnSO in the catalytic Ozonation of CIP

\begin{tabular}{llll}
\hline Reuse time & $\begin{array}{l}\text { CIP } \\
\text { degradation }^{a}(\%)\end{array}$ & $\begin{array}{l}\text { TOC } \\
\operatorname{removal}^{b}(\%)\end{array}$ & $c(\mathrm{Mn})^{a} / \mathrm{mg} \mathrm{L}^{-1}$ \\
\hline 0 & 92.1 & 60.2 & 0.097 \\
1 & 90.4 & 58.9 & 0.088 \\
2 & 89.7 & 57.8 & 0.076 \\
3 & 89.1 & 57.3 & 0.069
\end{tabular}

${ }^{a}$ Reaction time is $30 \mathrm{~min} .{ }^{b}$ Reaction time is $60 \mathrm{~min}$. produced during the process of catalytic ozonation decomposition. The reaction rate constant of TBA and hydroxyl radicals was $6.0 \times 10^{8} \mathrm{M}^{-1} \mathrm{~S}^{-1}$, which was much higher than the reaction rate constant of $3.0 \times 10^{-3} \mathrm{M}^{-1} \mathrm{~S}^{-1}$ for TBA and ozone. ${ }^{12}$ As shown in Fig. S6, $\uparrow$ the effects of TBA on the degradation of CIP were investigated. After the addition of TBA $\left(100 \mathrm{mg} \mathrm{L}^{-1}\right)$, the CIP degradation was significantly inhibited. When $100 \mathrm{mg} \mathrm{L}^{-1}$ of TBA was added into the aqueous solution, reductions of $54.5 \%$ and $28.9 \%$ for the degradation efficiency during the processes of catalytic ozonation and ozonation alone at $30 \mathrm{~min}$ were noted, respectively. It was shown that during the process of degradation of CIP, TBA and CIP competition for $\cdot \mathrm{OH}$ produced intermediates of high selectivity and inertia, ending the chain reaction of free radicals. ${ }^{41}$ This hindered the reaction of $\cdot \mathrm{OH}$ and CIP, resulting in a reduction in the CIP degradation rate in the system. At the same time, it was also indirectly proven that the catalytic ozonation degradation of the CIP system by MnSO was the mechanism of hydroxyl radical action.

To further explore the reaction mechanism, $\mathrm{O}_{3}$ was replaced by the $\mathrm{N}_{2}$ in same experimental setup. As shown in Fig. $\mathrm{S} 7, \dagger$ under the $\mathrm{MnSO} / \mathrm{N}_{2}$ system, the removal of CIP was $18.2 \%$. When $100 \mathrm{mg} \mathrm{L}^{-1} \mathrm{TBA}$ was added to the $\mathrm{MnSO} / \mathrm{N}_{2}$ system, the removal of CIP remained unchanged. It indicated that there was no $\cdot \mathrm{OH}$ in the water, and the removal of CIP was probably due to the adsorption of catalyst. In comparison, $\mathrm{MnCO} / \mathrm{O}_{3}$ system had significantly higher CIP removal. However, the addition of TBA greatly inhibited the degradation of CIP in $\mathrm{MnCO} / \mathrm{O}_{3}$ system. Base on above experiment results, it can be concluded that $\mathrm{O}_{3}$ decomposition was the sole source of $\cdot \mathrm{OH}$ under MnSO catalytic ozonization.

3.8.2 Mechanisms of catalytic ozonation by MnSO. From the aforementioned discussion, it was quite obvious that MnSO showed superior adsorption performance and perfect catalytic activity in heterogeneous catalytic ozonation to remove CIP. The oxidation reaction on the surface of the catalyst was the most important step in catalytic ozonation. When ozone or organic molecules were adsorbed on the catalyst surface, the oxidation reaction was significantly accelerated. To illustrate the possible interaction relation of ozone and the MnSO catalyst in the ozonation of CIP and its intermediates, a simplified mechanism showing the catalytic ozonation of CIP with the MnSO catalyst is shown in Fig. 8. Ozone can directly attack CIP in aqueous solution to generate $\mathrm{CO}_{2}$ and $\mathrm{H}_{2} \mathrm{O}$, but the rate of direct reaction is much less than that of the oxidation of the hydroxyl radicals.

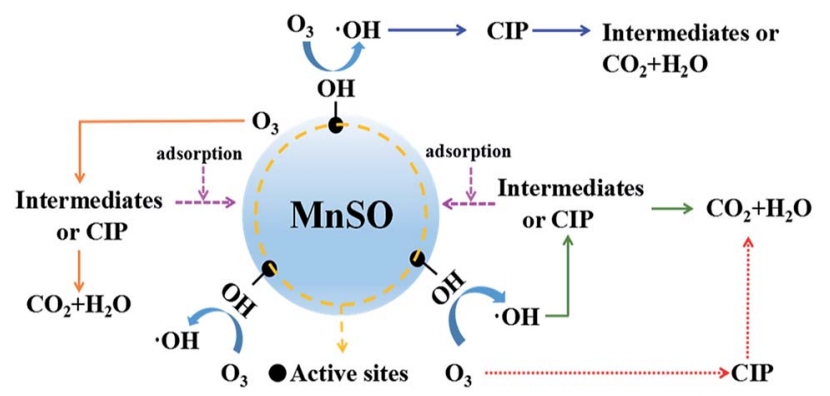

Fig. 8 Reaction mechanism of the MnSO process. 
The existence of $\cdot \mathrm{OH}$ resulted in successive oxidation reactions of CIP and its intermediates and promoted TOC removal.

The chain reactions of ozone decomposition were as followed:19

$$
\begin{gathered}
\mathrm{O}_{3}+\mathrm{H}_{2} \mathrm{O} \rightarrow 2 \cdot \mathrm{OH}+\mathrm{O}_{2}, k_{2}=1.1 \times 10^{-4} \mathrm{M}^{-1} \mathrm{~s}^{-1} \\
\mathrm{O}_{3}+\mathrm{OH}^{-} \rightarrow \mathrm{O}_{2}^{\cdot-}+\mathrm{HO}_{2}^{\cdot}, k_{2}=70 \mathrm{M}^{-1} \mathrm{~s}^{-1} \\
\mathrm{O}_{3}+\cdot \mathrm{OH} \rightarrow \mathrm{O}_{2}+\mathrm{HO}_{2}^{\cdot} \leftrightarrow \mathrm{O}_{2}^{\cdot-}+\mathrm{H}^{+} \\
\mathrm{O}_{3}+\mathrm{HO}_{2}^{\cdot} \rightarrow 2 \mathrm{O}_{2}+\cdot \mathrm{OH}, k_{2}=1.6 \times 10^{9} \mathrm{M}^{-1} \mathrm{~s}^{-1} \\
2 \mathrm{HO}_{2}^{\cdot} \rightarrow \mathrm{O}_{2}+\mathrm{H}_{2} \mathrm{O}_{2}
\end{gathered}
$$

Furthermore, the adsorption of CIP and the intermediates on the MnSO catalyst contributed to the increase in the CIP and TOC removal, which is also beneficial to the subsequent oxidation process.

Consequently, in this study, there were three pathways for heterogeneous catalytic ozonation as follows: ${ }^{41}$ (1) chemisorption of ozone on the catalyst surface was decomposed by the formation of surface oxygenated radical species, which promoted the formation of hydroxyl radicals. The hydroxyl radicals rapidly removed CIP and the intermediates. (2) Organic molecules (CIP or intermediates) adsorbed on the surface of the catalyst were oxidized by gaseous or aqueous ozone. (3) Adsorption of both ozone and organic molecules (CIP or intermediates) on the surface of the MnSO catalyst and their direct and indirect reactions occurred between ozone and CIP, leading to a rapid degradation of CIP.

The reactions of CIP degradation can be simplified as: ${ }^{\mathbf{4 4}}$

(I). Indirect (radical) reactions.

(a). Catalyst surface reaction:

$$
\begin{gathered}
\mathrm{MnSO}^{-\cdot \mathrm{OH}}+\mathrm{CIP} \rightarrow \mathrm{MnSO}+\mathrm{CO}_{2}+\mathrm{H}_{2} \mathrm{O}+\text { intermediates } \\
\mathrm{MnSO}^{-\cdot \mathrm{CIP}}+\cdot \mathrm{OH} \rightarrow \mathrm{MnSO}+\mathrm{CO}_{2}+\mathrm{H}_{2} \mathrm{O}+\text { intermediates }
\end{gathered}
$$

(b). Bulk solution reaction:

$$
\mathrm{CIP}+\cdot \mathrm{OH} \rightarrow \text { intermediates }+\mathrm{CO}_{2}+\mathrm{H}_{2} \mathrm{O}
$$

(II). Direct (molecular $\mathrm{O}_{3}$ ) reactions.

(a). Catalyst surface reaction:

$$
\begin{gathered}
\mathrm{MnSO}^{-\cdot \mathrm{O}_{3}}+\mathrm{CIP} \rightarrow \mathrm{MnSO}+\mathrm{CO}_{2}+\mathrm{H}_{2} \mathrm{O}+\text { intermediates } \\
\mathrm{MnSO}^{-\cdot} \mathrm{CIP}+\mathrm{O}_{3} \rightarrow \mathrm{MnSO}+\mathrm{CO}_{2}+\mathrm{H}_{2} \mathrm{O}+\text { intermediates }
\end{gathered}
$$

(b). Bulk solution reaction:

$$
\mathrm{CIP}+\mathrm{O}_{3} \rightarrow \text { intermediates }+\mathrm{CO}_{2}+\mathrm{H}_{2} \mathrm{O}
$$

In addition, according to the catalyst characterization results shown in Fig. 2 and S2, $\dagger$ different states of manganese on the surface of MnSO led to more electron transfer opportunities and promoted its high catalytic activity. ${ }^{45}$

\section{Conclusions}

Manganese modified natural SO catalysts were successfully prepared using the impregnation method and showed remarkable catalytic activity for the degradation and mineralization of CIP. The characterizations of the MnSO catalyst showed that $\mathrm{MnO}_{x}$ was distributed on the surface of the SO. The CIP removal rate with an MnSO catalyst at $30 \mathrm{~min}$ could reach 92.1\%, but just 53\% using $\mathrm{O}_{3}$ alone. The removal rate of TOC was $30.4 \%$ higher during the catalytic ozonation than with $\mathrm{O}_{3}$ alone. During MnSO catalytic ozonation, with the increase in catalyst dosage and the initial solution $\mathrm{pH}$, the CIP and TOC removal efficiency in aqueous solution both increased. However, the capability of the catalyst for CIP degradation and TOC removal decreased with an increase in the initial concentration of CIP. The MnSO catalytic ozonation followed the $\cdot \mathrm{OH}$ production mechanism. The CIP removal was attributed to a synergistic effect of the adsorption, ozone oxidation, and $\cdot \mathrm{OH}$ oxidation. The activity of MnSO on CIP degradation and mineralization slightly decreased with repeated use several times. This was because of the leaching of the manganese ion from MnSO into solution, while the MnSO catalyst maintained excellent activity. This study may provide information on an inexpensive, viable, and promising catalyst towards effective catalytic ozonation of antibiotics from wastewater for practical application.

\section{Conflicts of interest}

There are no conflicts to declare.

\section{Acknowledgements}

This work was financially supported by the National Science and Technology Major Projects for Water Pollution Control and Treatment (Grant No. 2017ZX07201003); Natural Science Foundation and Technology Department of Jilin Province, China (20150101093C); and Technology Department Key Research of Jilin Province, China (20140204038SF).

\section{References}

1 D. Li, X. Guo, H. Song, T. Sun and J. Wan, J. Hazard. Mater., 2018, 180, 957.

2 T. Paul, P. L. Miller and T. J. Strathmann, Environ. Sci. Technol., 2007, 41, 4720.

3 H. Xiong, S. Dong, J. Zhang, D. Zhou and B. E. Rittmann, Water Res., 2018, 136, 75.

4 H. Nakata, K. Kanaan, P. D. Jones and J. P. Giesy, Chemosphere, 2005, 58, 759.

5 D. W. Kolpin, E. T. Furlong, M. T. Meyer, E. M. Thurman, S. D. Zaugg, L. B. Barber and H. T. Buxton, Environ. Sci. Technol., 2002, 36, 1202.

6 D. G. Larsson, P. C. De and N. Pedro, J. Hazard. Mater., 2007, 148, 751.

7 P. Zheng, B. Bai, W. S. Guan, H. L. Wang and Y. R. Suo, RSC Adv., 2016, 6, 4101. 
8 B. Kasprzyk-Hordern, M. Ziółek and J. Nawrocki, Appl. Catal., $B, 2003,46,639$.

9 J. Nawrockia and B. Kasprzyk-Hordern, Appl. Catal., B, 2010, 99, 27.

10 L. Yang, C. Hu, Y. Nie and J. Qu, Appl. Catal., B, 2010, 97, 340.

11 H. M. Jalali, Mater. Sci. Eng., C, 2016, 59, 924.

12 B. Roshani, I. Mcmaster, E. Rezaei and J. Soltan, Sep. Purif. Technol., 2014, 135, 158.

13 F. Nawaz, H. Cao, Y. Xie, J. Xiao, Y. Chen and Z. A. Ghazi, Chemosphere, 2017, 168, 1457.

14 R. Andreozzi, A. Insola, V. Caprio, R. Marotta and V. Tufano, Appl. Catal., A, 1996, 138, 75.

15 J. Ma, M. Sui, T. Zhang and C. Guan, Water Res., 2005, 39, 779.

16 M. Sui, S. Xing, L. Sheng, S. Huang and H. Guo, J. Hazard. Mater., 2012, 227-228, 227.

17 W. Chen, X. Li, Z. Pan, S. Ma and L. Li, Sep. Purif. Technol., 2017, 173, 99.

18 M. Sui, J. Liu and L. Sheng, Appl. Catal., B, 2011, 106, 195.

19 R. Huang, H. Yan, L. Li, D. Deng, Y. Shu and Q. Zhang, Appl. Catal., B, 2011, 106, 264.

20 X. Lu, Q. Zhang, W. Yang, X. Li, L. Zeng and L. Li, RSC Adv., 2015, 14, 10537.

21 F. Qi, B. Xu, L. Zhao, Z. Chen, L. Zhang, D. Sun and J. Ma, Appl. Catal., B, 2012, 121-122, 171.

22 G. Moussavi, R. Khosravi and N. R. Omran, Appl. Catal., A, 2012, 445, 42.

23 K. He, Y. Dong, L. Yin, A. Zhang and Z. Li, Acta Geochim., 2006, 25, 587.

24 J. S. Park, H. Choi and J. Cho, Water Res., 2004, 38, 2284.

25 N. Sano, T. Yamamoto, D. Yamamoto, S. I. Kim, A. Eiad-Ua, H. Shinomiya and M. Nakaiwa, Chem. Eng. Process., 2007, 46, 513.

26 H. Zhang, F. Ji, Y. Zhang, Z. Pan and B. Lai, Sep. Purif. Technol., 2017, 193, 368.
27 F. Arena, G. Trunfio, J. Negro, B. Fazio and L. Spadaro, ChemInform, 2007, 38, 2269.

28 C. K. Sang and G. S. Wang, Appl. Catal., B, 2010, 98, 180.

29 B. M. Reddy, B. Chowdhury and P. G. Smirniotis, Appl. Catal., A, 2001, 211, 19.

30 W. Wang, J. Yu, J. Zou and X. Yu, Electrochim. Acta, 2016, 191, 426.

31 D. Li, T. Sun, L. Wang and N. Wang, Electrochim. Acta, 2018, 282, 416.

32 X. Tan, Y. Wan, Y. Huang, C. He, Z. Zhang, Z. He, L. Hu, J. Zeng and D. Shu, J. Hazard. Mater., 2017, 321, 162.

33 H. Yan, P. Lu, Z. Pan, X. Wang, Q. Zhang and L. Li, J. Mol. Catal. A: Chem., 2013, 377, 57.

34 A. D. Kiadehi, A. Ebadi and A. Aghaeinejad-Meybodi, Sep. Purif. Technol., 2017, 182, 238.

35 Y. Xu, H. Bai, G. Lu, C. Li and G. Shi, J. Am. Chem. Soc., 2008, 130, 5856.

36 Z. Ji, X. Shen, J. Yang, G. Zhu and K. Chen, Appl. Catal., B, 2014, 144, 454.

37 S. P. Tong, W. P. Liu, W. H. Leng and Q. Q. Zhang, Chemosphere, 2003, 50, 1359.

38 S. P. Ghuge and A. K. Saroha, J. Environ. Manage., 2018, 211, 83.

39 Z. Liu, J. Tu, Q. Wang, Y. Cui, L. Zhang, X. Wu, B. Zhang and J. Ma, Sep. Purif. Technol., 2018, 200, 51.

40 C. A. Orge, J. J. M. Orfao, M. F. R. Pereira, A. M. D. D. Farias, R. C. R. Neto and M. A. Fraga, Appl. Catal., B, 2011, 103, 190.

41 L. Zhao, J. Ma, Z. Sun and H. Liu, Appl. Catal., B, 2009, 89, 326.

42 T. Zhang, J. Mol. Catal. A: Chem., 2008, 279, 82.

43 Ministry of Health of the People's Republic of China, Standards for drinking water quality (GB5749-2006), 2006.

44 G. Moussavi, A. A. Aghapour and K. Yaghmaeian, Chem. Eng. J., 2014, 249, 302.

45 Y. Huang, Y. Sun, Z. Xu, M. Luo, C. Zhu and L. Li, Sci. Total Environ., 2017, 575, 50. 